\title{
Les dépôts de matériel moteur ferroviaire de la région Tourangelle
}

The railway engine equipment depots of the Tours region

Jean Metz et Yves Machefert-Tassin

\section{OpenEdition}

\section{Journals}

Édition électronique

URL : https://journals.openedition.org/rhcf/1790

DOI : $10.4000 /$ rhcf. 1790

\section{Éditeur}

Rails \& histoire

\section{Édition imprimée}

Date de publication : 1 décembre 2003

Pagination : $375-389$

ISBN : 0996-9403

ISSN : 0996-9403

\section{Référence électronique}

Jean Metz et Yves Machefert-Tassin, «Les dépôts de matériel moteur ferroviaire de la région

Tourangelle », Revue d'histoire des chemins de fer [En ligne], 28-29 | 2003, mis en ligne le 31 décembre 2014, consulté le 22 avril 2022. URL : http://journals.openedition.org/rhcf/1790 ; DOI : https://doi.org/ $10.4000 /$ rhcf. 1790 


\section{Les dépôts de matériel moteur ferroviaire de la région Tourangelle}

La région tourangelle et la ville de Tours en particulier furent desservies de très bonne heure par le chemin de fer, puisque «l'embarcadère » a été ouvert le 2 avril 1846 au service des voyageurs, trois ans seulement après Paris-Orléans, et, à l'époque, des villes telles que Dijon, Toulouse ou Nancy, pour n'en citer que quelques-unes, ne connaissaient pas le chemin de fer. Tours, dès cette époque, reçut ainsi son premier dépôt de locomotives (Compagnie d'Orléans à Bordeaux), à savoir une modeste rotonde complète, et une dotation d'une douzaine de machines. En effet, la fusion des compagnies de chemin de fer en grands réseaux n'était pas encore accomplie et Tours a initialement connu une desserte par plusieurs compagnies, chacune disposant de ses propres installations pour les locomotives de ses propres lignes, à savoir :

- celle d'Orléans à Bordeaux, en 1846 (alors indépendante du Paris-Orléans);

- celle de Tours à Nantes en 1848,

lesquelles se joignirent à :

- celle de Paris à Orléans (datant de 1843)

et devinrent le $\mathrm{PO}$ en 1852 après la fusion des compagnies initiales.

Elles réunissent alors sous l'égide du $\mathrm{PO}$ les compagnies du Centre, d'Orléans à Bordeaux et de Tours à Nantes.

S'ajoute ensuite celle de la Vendée, en 1875, avec des installations de traction et ateliers totalement séparés des précédentes. Elles devient le réseau de l'État en 1878 après la reprise de la Vendée par l'État qui réunit au sud de la Loire plusieurs compagnies dont les plus importantes sont la Vendée et la Charente.

À côté du chemin de fer à voie normale, le département bénéficie dès 1884 d'un réseau secondaire à voie métrique exploité par la Compagnie des chemins de fer départementaux (CFD) et l'agglomération de Tours d'un réseau de tramways, à chevaux dès 1877 , puis à vapeur en 1889 et électrique dès 1899 .

Cette multiplicité d'exploitants ferroviaires entraîna au XIX ${ }^{e}$ siècle un grand développement des installations du chemin de fer avec pour corollaire une population cheminote importante : après avoir compté 
jusqu'à 6000 cheminots et quatre dépôts totalisant moins de 100 machines dans la première moitié du $\mathrm{xx}^{\mathrm{e}}$ siècle, la $\mathrm{SNCF}$, héritière des compagnies avec environ 2000 emplois, représente encore actuellement le premier employeur de la région tourangelle. Les installations gèrent plus de 250 engins de traction. Sur le plan des infrastructures ferroviaires, depuis plus de 150 ans Tours et Saint-Pierre-des-Corps forment un ensemble important : encore huit lignes et une ligne TGV y aboutissent, nécessitant des installations pour les voyageurs avec les gares de Tours et Saint-Pierre-des-Corps, une gare de triage pour les marchandises, un dépôt pour les locomotives et automotrices et un grand atelier de maintenance pour l'entretien des automotrices et du matériel remorqué, l'EIMM (établissement industriel de maintenance de matériel) de Saint-Pierre-des-Corps.

Dans le domaine plus spécifique de la traction, hors les grands ateliers généraux, Tours - Saint-Pierre-des-Corps a compté jusqu'à sept dépôts d'engins moteurs à voie normale, auxquels il faut ajouter pour la région tourangelle les deux dépôts à voie métrique des CFD et les deux dépôts de tramways, l'un à vapeur à Vouvray, l'autre électrique à Tours. De nos jours ne subsiste plus que le dépôt de Saint-Pierre-desCorps de la SNCF, ou « Tours-Saint-Pierre ».

\section{Situation et missions des dépôts}

Sur la commune de Tours cinq sites ont existé, dont certains simultanément (par exemple OB, TN et Vendée). Le dépôt de la Compagnie d'Orléans à Bordeaux, $\mathrm{OB}$, composé d'une rotonde à 16 voies avec plaque tournante de $9 \mathrm{~m}$ puis $12 \mathrm{~m}$ au centre, est complété dès 1851 par une rotonde annulaire de neuf places, toutes équipées de ponts ou plaques de $12 \mathrm{~m}$. La remise annulaire est située à droite des voies en arrivant d'Orléans, juste avant la passerelle à piétons qui enjambe les voies et la zone d'ateliers de la rotonde $O B$. Un atelier en $U$ comportant les magasins, la chaudronnerie pour les chaudières en cuivre, les forges et la fonderie complétait un atelier d'ajustage et montage, avec machine à vapeur fixe entrainant la tournerie et les machines outils. La Compagnie de Tours à Nantes disposait d'une rotonde circulaire identique à celle de l'OB, à 16 places, mais n'avait comme bâtiments pour les ateliers de locomotives que le tiers de la surface dont disposait l'OB. Les ateliers de Tours à Nantes inachevés étaient prévus pour être construits symétriquement à ceux de l'OB par rapport au tronc commun des voies. 
Ce dépôt de la Compagnie de Tours à Nantes, TN, presque identique et symétrique à celui de l'OB et situé à gauche des voies en arrivant de Nantes, jouxte les ateliers principaux qui deviendront ceux du PO (voir dans ce volume la communication de B. Monteil et C. Colombier). Ces deux premières rotondes étaient bâties en maçonnerie avec charpente en fer.

Il faut remarquer qu'à cette époque les compagnies ne faisaient pas de distinction entre atelier de dépôt et grands ateliers ; ceux du TN, plus petits à l'origine que ceux de l'OB, étaient les seuls à permettre les agrandissements ultérieurs d'après 1900. En effet, très rapidement les installations de l'OB furent trop petites et dès 1880 il fallut envisager de parquer plus de 60 unités (fig. 1).

Pour créer le dépôt de la Compagnie de Paris à Orléans après la fusion de 1852 le PO reprend les installations existantes mais séparées de l'OB et du TN et agrandit d'abord la remise annulaire est de neuf places pour en faire une demi-circonférence à 15 places. Dès 1860, on envisage de séparer dépôt et grands ateliers. L'idée était d'intégrer la rotonde TN aux ateliers du matériel moteur et remorqué, à l'ouest des voies. En 1890 les installations primitives sont devenues insuffisantes et le $\mathrm{PO}$ construit un nouveau dépôt à remise de type rectangulaire à transbordeur incorporé pouvant offrir jusqu'à 48 places abritées, en trois nefs, toujours existantes, susceptibles d'être doublées. Puis, après 1917, une rotonde à 14 voies couvertes, une rotonde à découvert et trois voies d'entrée et sortie s'y rajoutent. Le dépôt de combustibles des années 1910 est installé en prolongement du pont.

Ce dépôt se trouvait dans le triangle formé par les voies de Saint-Pierre-des-Corps à Tours, de Tours à Bordeaux et le raccordement de Saint-Pierre à Nantes. Il est construit en maçonnerie avec charpente métallique et des stalles en béton armé (fig. 2$)^{1}$.

Progressivement l'ancienne remise de 1880 sera utilisée comme atelier du dépôt.

De l'origine à 1875 les dépôts $\mathrm{PO}$ abritèrent des locomotives de vitesse à disposition d'essieux 111 ou 120 et des machines mixtes ou à marchandises à disposition d'essieux 120, 021 et 030 . Toutes ces machines roulaient de Tours à Orléans, à Poitiers et Angoulême, à Angers et Nantes, au Mans, à Châteaudun, à Vierzon et à Bourges. Le dépôt PO de Tours se trouvait sensiblement au centre du réseau entre Paris et Bordeaux d'une part, Saincaize et Saint-Nazaire d'autre part.

1- Le Patrimoine des chemins de fer, Charenton, Flohic éditions, 2000 ( $2^{\mathrm{e}}$ éd. ), p. 281. 


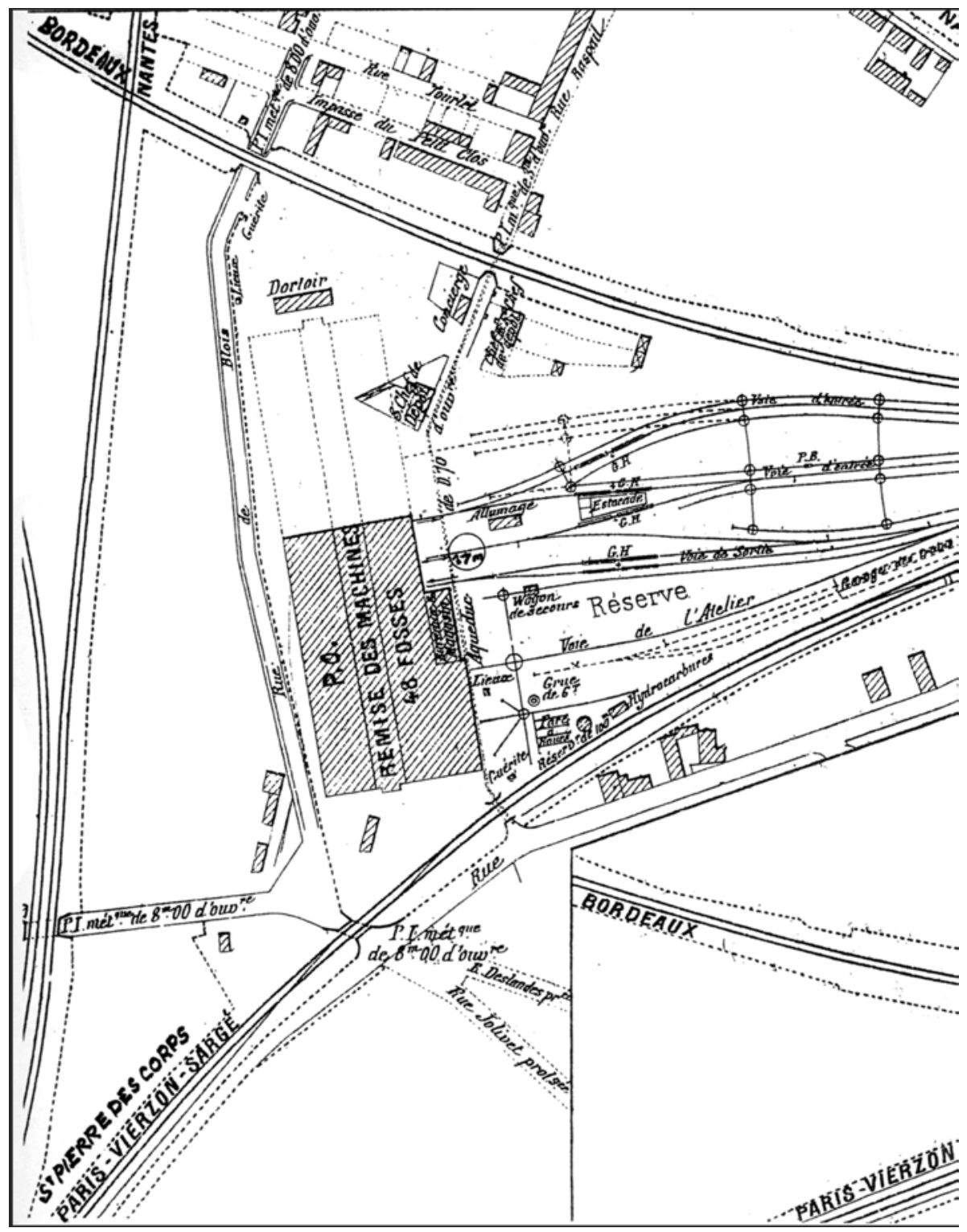

Figure 1. Les dépôts de Tours $\mathrm{OB}, \mathrm{TN}$ et $\mathrm{PO}$ avant 1898. En bas, les dépôts à rotondes d'origine $\mathrm{OB}$ et $\mathrm{TN}$, de part et d'autre des voies vers la gare terminus de Tours. En haut le dépôt $\mathrm{PO}$ à remise rectengulaire à 3 halles dont, au centre, le transbordeur de desserte des 48 fosses. Source : Le Génie civil, 1901. 
Jean METZ / Yves MACHEFERT-TASSIN

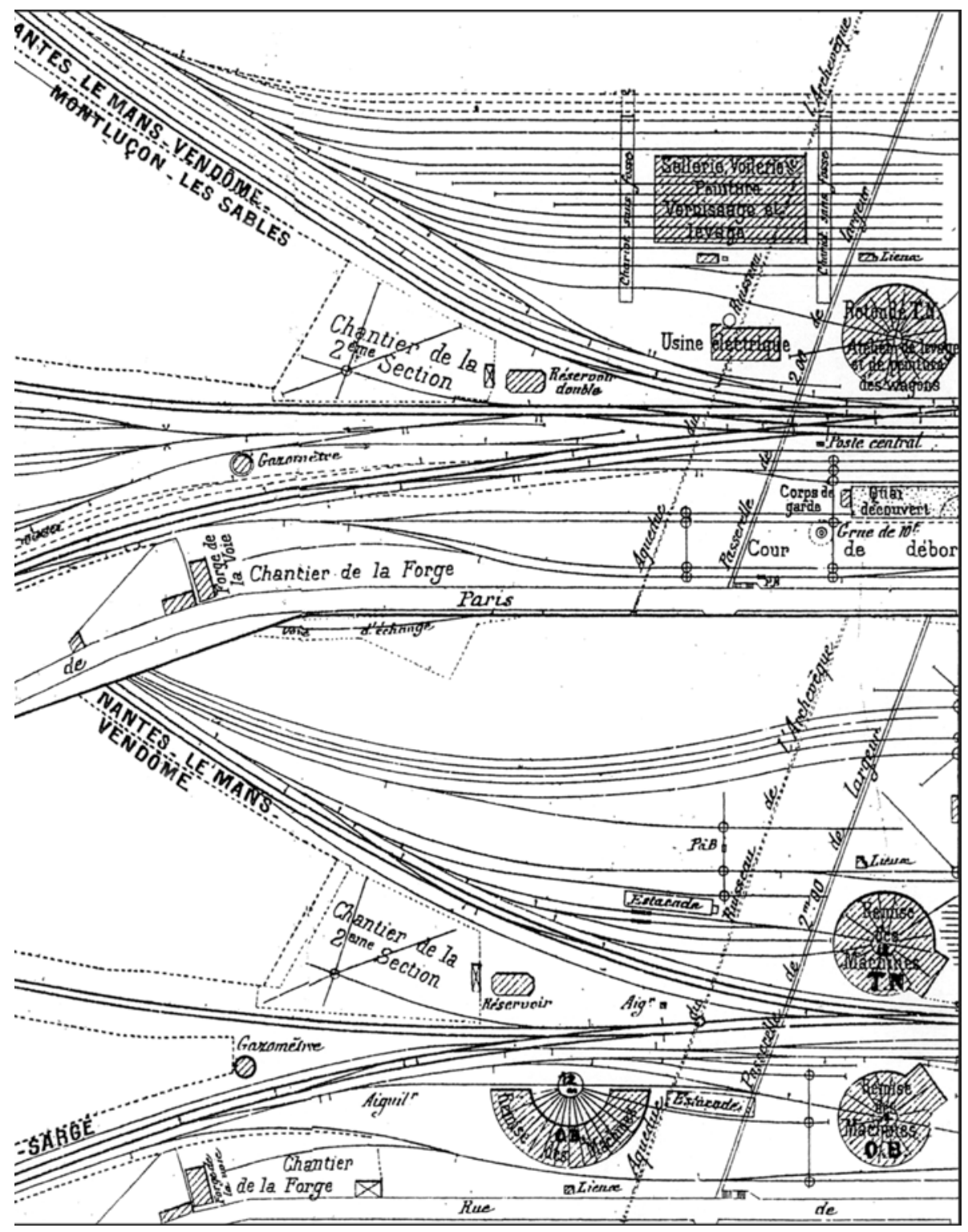




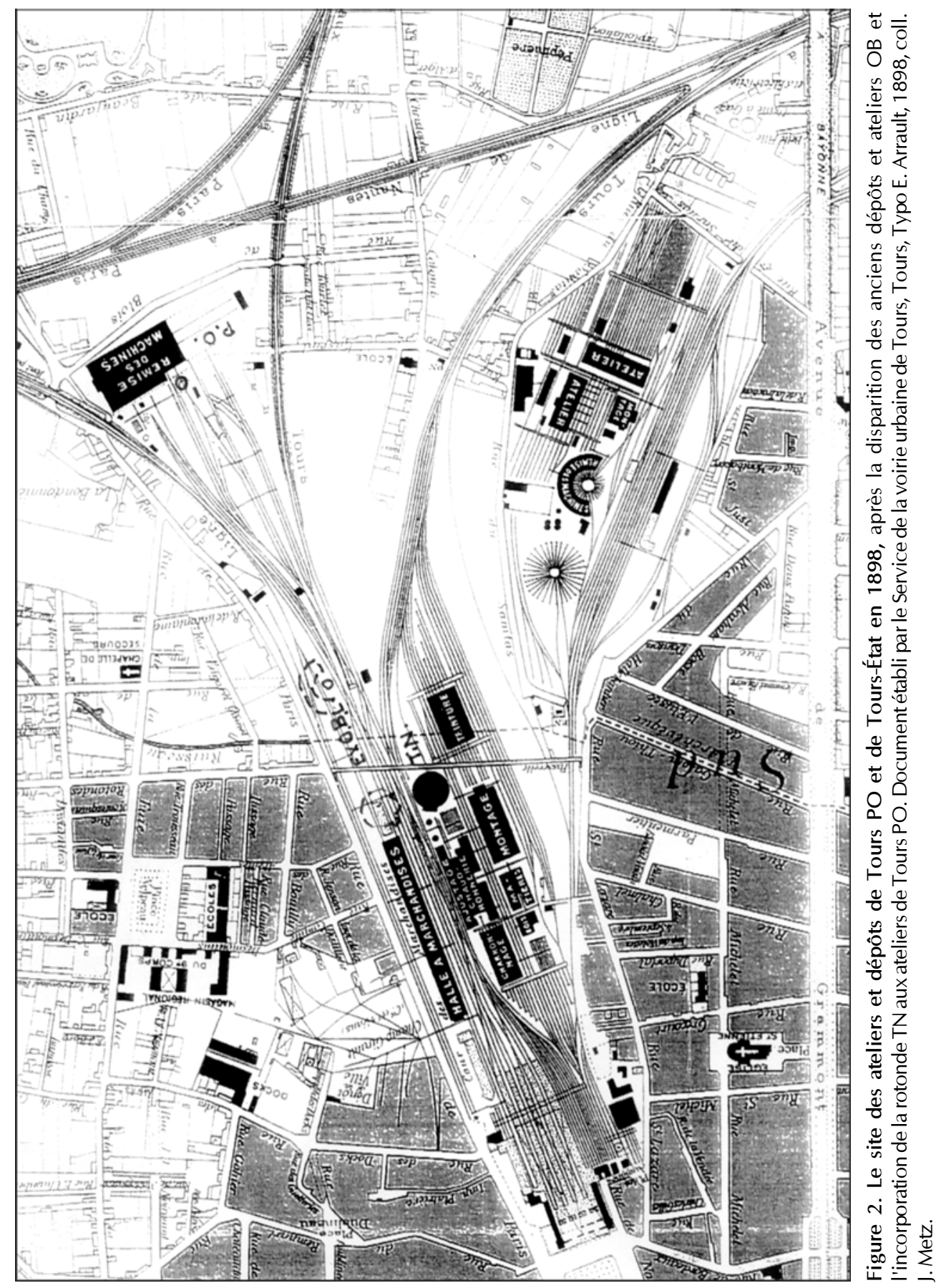




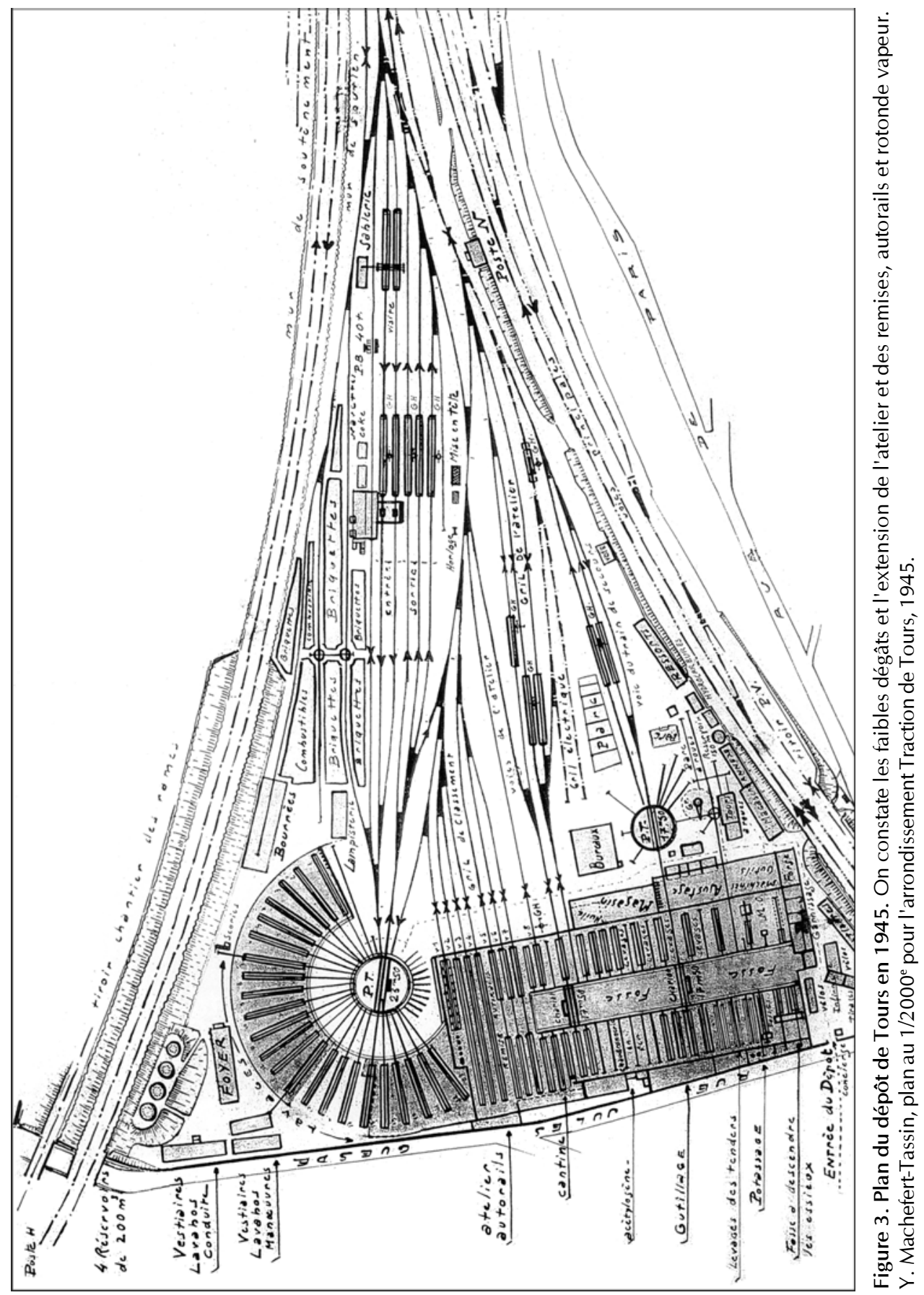


En 1875 l'arrivée à Tours de la ligne de la Vendée venant des Sables-d'Olonne par Bressuire et Thouars entraine l'ouverture d'un nouveau dépôt, celui de la Compagnie de la Vendée qui était concessionnaire, en plus de la ligne des Sables à Tours, de la ligne de Tours à Montluçon par Châteauroux. Ce dépôt est décrit plus loin. La croissance du parc de locomotives de Tours est tel que, dès 1900, plus de 80 machines peuvent demeurer au dépôt, avec 64 places sur fosses, pour atteindre 125 locomotives en 1930, puis une réduction suivra les électrifications. Ainsi le déclin sera de 116 vapeurs en 1936 à 80 en 1940, Saint-Pierre restant à 60-65 machines. Le bâtiment de l'atelier du dépôt PO, converti en atelier commercial, existe toujours en 2003, ainsi que le bureau de la feuille (mouvement) (fig. 3 et RHCF no 18, p. 29).

Le dépôt de la Compagnie de la Vendée puis de l'État, créé en 1875 lors de l'ouverture de la ligne des Sables-d'Olonne à Tours par la Compagnie de la Vendée, devint propriété de l'État en 1878. Situé à l'ouest des voies PO, sur la commune de Tours, il s'étendait sur près de 20 ha avec ses ateliers entre les rues de Sanitas et du Hallebardier, au nord des ateliers généraux de la Vendée. Il se composait d'une remise annulaire sur environ les deux tiers de la circonférence avec 21 voies couvertes, 14 découvertes, d'un parc extérieur desservi par un pont tournant de $20 \mathrm{~m}$ et comptant 31 voies plus deux voies d'accès. Au sud plusieurs autres bâtiments d'ateliers généraux desservis par des ponts transbordeurs permettaient les travaux d' entretien du matériel tant moteur que remorqué. Sauf la rotonde, les ateliers (devenus Billard) ont survécu jusque dans les années 1960 (fig. 4).

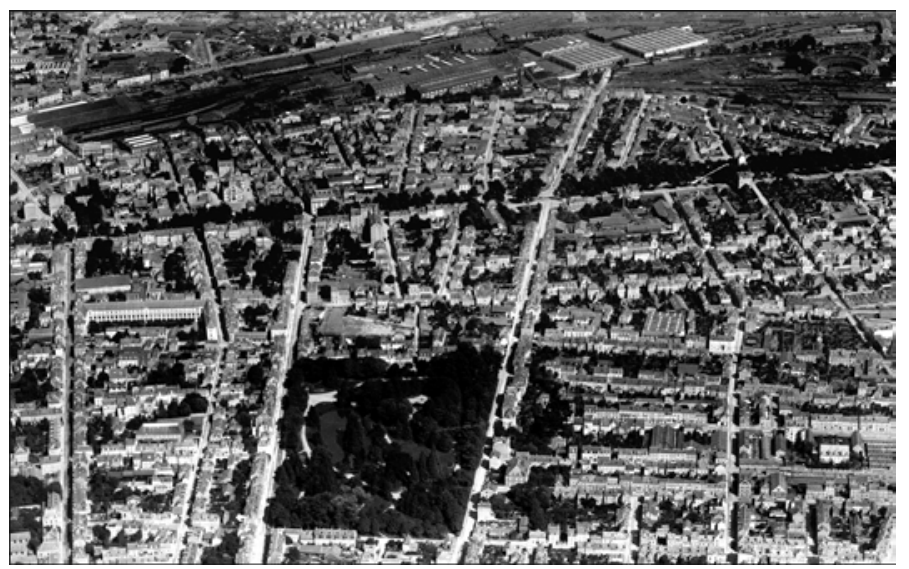

Figure 4. Photographie aérienne des ateliers PO et du dépôt État vus vers le sud-est de la gare de Tours vers la rotonde annulaire "Vendée ", 1920. $\mathrm{Cl}$. Capitaine Metz, base aérienne de Tours, coll. J. Metz. 
À Saint-Pierre-des-Corps, le développement du triage depuis 1890 entraina la création par le PO d'un dépôt près du triage pour éviter les mouvements de locomotives à marchandises entre le dépôt de Tours et les installations de Saint-Pierre. Après un changement de site, autorisé en 1907, le dépôt de Saint-Pierre entra en fonction au début de 1909 et se comporta en établissement indépendant, si l'on excepte la mise en commun du parc à combustibles. Le PO important une partie de son charbon d'Angleterre par Nantes, un important parc à charbon fut construit à Saint-Pierre-des-Corps. Ce parc fournissait bon nombre de dépôts tels Blois, Orléans, Vierzon, Bourges, etc.

Le dépôt était composé jusqu'en 1956 d’une demi-rotonde (quatre puis 18 places) avec pont tournant de $23,50 \mathrm{~m}$, située à droite des voies principales en venant d'Orléans avant d'arriver à la gare voyageurs de Saint-Pierre et joint au triage par un saut de mouton à deux voies vers le milieu du dépôt. Démoli en partie par les bombardements de 1944, il sera réutilisé puis ensuite reconstruit vers 1957-1960 en plus grand pour devenir l'actuel dépôt SNCF de Tours - Saint-Pierre-des-Corps, concentrant ainsi les installations de traction (fig. 5).

En 1917 l'arrivée des troupes américaines en France par le port de Nantes-Saint-Nazaire et Bordeaux ou La Rochelle entraina une très forte augmentation des transports, tant en hommes de troupe qu'en matériels. Les Américains amenèrent avec eux des locomotives et des wagons. L' axe principal d'acheminement de Nantes à Is-sur-Tille passe par Tours, Vierzon, Bourges, Saincaize, Dijon. Il fut nécessaire de construire des installations ferroviaires supplémentaires à celles existantes à Saint-Pierre.

À La Riche, près de Tours au lieu dit Saint-Cosme, furent ainsi créés un triage et un dépôt de relais (le principal étant à Gièvres : voir RHCF $\mathrm{n}^{\circ} 18$, p. 75), des ateliers de forge, mécanique et chaudronnerie.

À Tours, avenue de Grammont, près des lignes de Tours à Nantes et au Mans, se trouvait le dépôt des tramways de Tours, TT, composé d'une remise abritant 50 motrices et 60 remorques, d'un atelier de mécanique et d'électricité et d'une centrale thermique produisant le courant pour le réseau.

Ainsi, du fait de la disparition de la locomotive à vapeur, on a vu ces installations se transformer et se développer sur la commune de Saint-Pierre-des-Corps avec le nouveau dépôt très important et un atelier encore plus important, existant depuis le début du siècle, l'EIMM, qui traite l'entretien et la réparation de toutes les automotrices électriques du réseau SNCF. 


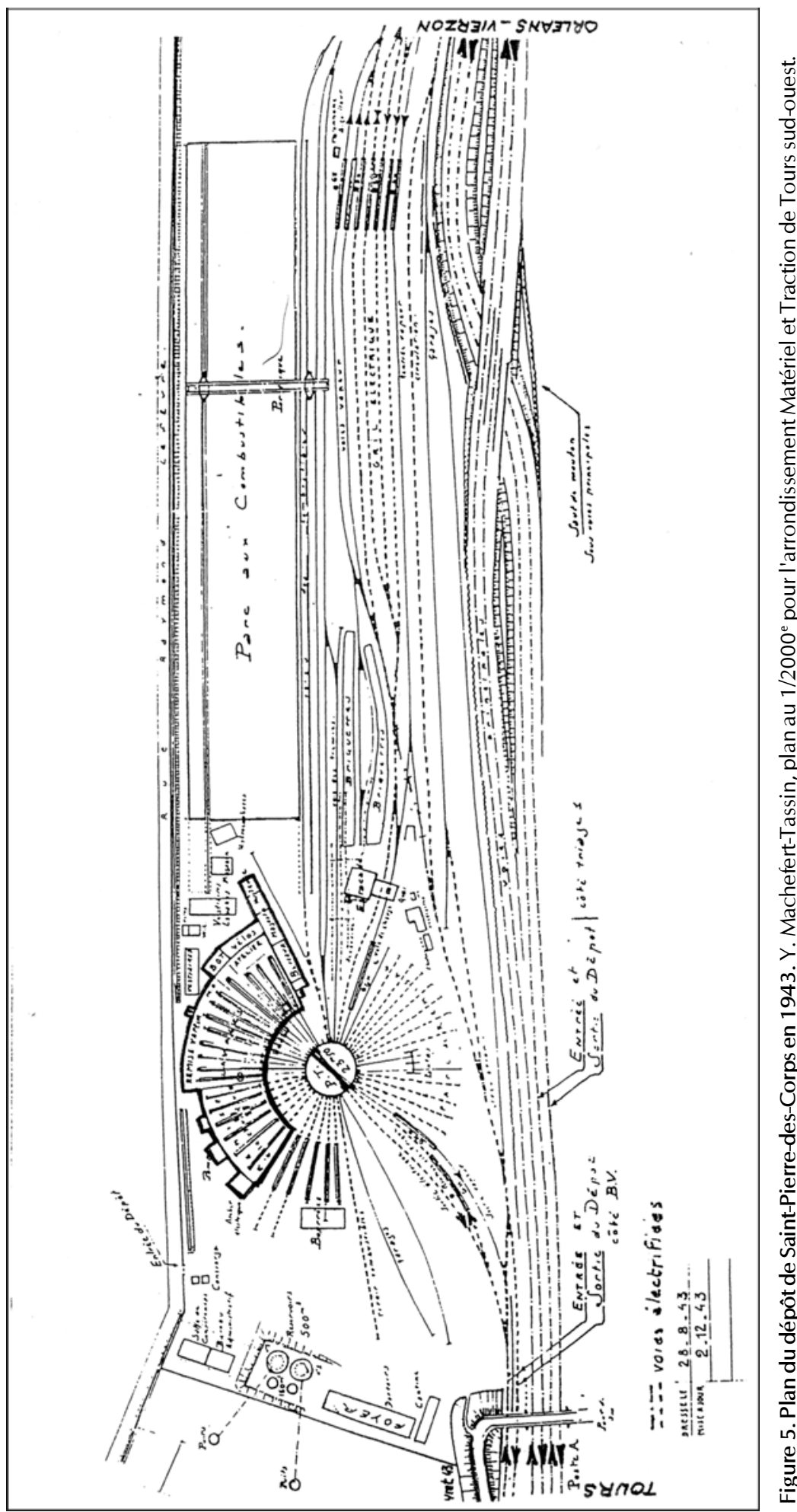


Rappelons que ce dernier fait suite à la longue tradition des ateliers de chemins de fer tourangeaux, dont ceux de Tours (locomotives et voitures) puis Saint-Pierre-des-Corps (voitures et wagons, fonderies, et magasins généraux de l'ancien réseau $\mathrm{PO}$ ), sans parler des activités industrielles intermédiaires issues d'ateliers convertis (Billard, CIMT, Cadoux, etc.).

\section{Regroupement et destructions-reconstructions à Tours - Saint-Pierre-des-Corps : PO, PO-MIDI, et SNCF}

Les descriptions de l'évolution des installations précédentes à voie normale montrent dès le début du siècle dernier des hésitations quant au choix du nouveau site du dépôt de Tours. En font foi les projets de communauté de dépôts de Tours (1894-1895) et de Saint-Pierre-desCorps (1895-1897). Cependant la séparation reste assez théorique, après 1907-1910, entre Saint-Pierre, spécialisé en locomotives marchandises, proches du triage, puis recevant ensuite (1933) les engins électriques, et Tours spécialisé en trafic voyageurs et messageries, d’abord à vapeur puis en autorails à partir de 1932. L'évolution envisagée par le PO semble bien être, dès les années 1930, un futur regroupement des locomotives, à vapeur et électriques, à Saint-Pierre, près du parc à combustibles, avec de nouvelles installations (environ 18 à 24 places de remisage en rotonde et atelier mixte pour quatre à six levages simultanés).

De son côté Tours devait être maintenu dans son rôle de dépôt autorails et automotrices électriques, vu la proximité de la gare Tours voyageurs, terminus originaire des neuf lignes en étoile à desservir avec ces engins automoteurs. En 1939-1940 on compte près de 120 locomotives à vapeur et 15 à 20 autorails à Tours et environ 50 locomotives à vapeur et une trentaine d'électriques à Saint-Pierre.

Cependant l'atelier du dépôt de Tours peut assurer de grosses révisions ou réparations du parc des deux dépôts, et Saint-Pierre depuis son origine (1910) assure l'approvisionnement en combustibles des deux établissements. La guerre ne modifie pas la répartition des rôles sauf par la diminution des mouvements d'engins de vitesse à vapeur à Tours, après l'électrification de Tours - Bordeaux (1938), et par le renfort correspondant de Saint-Pierre en locomotives et automotrices électriques, passant de 30 à 50, avec même quelques attributions de 2D2 (pour les messageries notamment). Après la destruction massive du triage de Saint-Pierre en avril 1944 restent cependant 12 places dans la remise annulaire en bois. À Tours on observe des dégâts relativement réduits sur les grands bâtiments, sauf la rotonde atteinte en mai 1944 
(fig. 6), ce qui permet de reprendre l'activité assez vite fin 1944 et début 1945. On retrouve des effectifs de machines proches de ceux de 1943, y compris le retour des autorails.

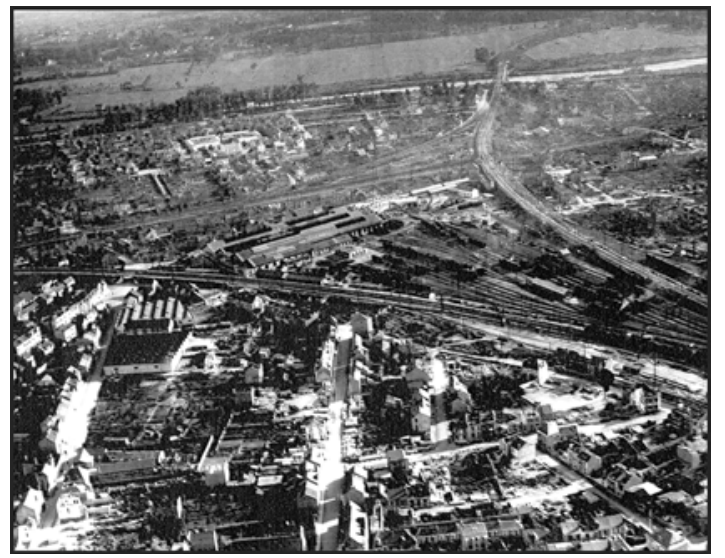

Figure 6. Vue aérienne du dépôt de Tours en 1946, commande de l'arrondissement Voie et Bâtiments de Tours à la base aérienne de Tours, prise vers le Sud. On distingue les bâtiments de l'atelier du dépôt, à gauche, et le reste de la rotonde annulaire, à droite, épargnés par les bombardement et remis en état. Par contre I'environnement civil n'est pas épargné.

Coll. Y. Machefert-Tassin.

Des plans de reconstruction et de regroupement des deux dépôts abondent, mais les services de la traction sud-ouest, au 41 boulevard de la gare à Paris XIII ${ }^{\mathrm{e}}$, ne soutiennent pas le projet de deux rotondes annulaires type « $\mathrm{P}$ » et d'un atelier « $\mathrm{A}$ » à Saint-Pierre, qu'ils jugent peu appropriés aux modes de traction à venir. Ils étaient en effet défendus par les services vapeur de l'ex - PLM, dont les études de dépôts résidaient gare de Lyon (voir la communication de N. Nogue).

Mais ces plans semblaient ignorer les projets sud-ouest des liaisons grande vitesse Paris-Tours avec des rames automotrices électriques, dites Ruban bleu (Alsthom) aptes à $180-200 \mathrm{~km} / \mathrm{h}$ et datant de 1938, de même que les développements de la traction Diesel, pour les locomotives ou autorails, amorcés avant guerre. Or il se trouve qu'après la guerre, grâce à R. Parès, l'ingénieur en chef de la Traction (M. Riou) a demandé à l'un de nous, Y. Machefert-Tassin, en stage à l'arrondissement Traction de Tours, une étude sur les évolutions possibles vers un dépôt commun de la traction et ses infrastructures dans l'arrondissement. La réponse conduisait à s'inspirer de ce qui avait été fait à Nantes-Blottereau, par le PO et l'Ouest, et à Béziers par le Midi, afin d'aboutir à un plan à voies et bâtiments parallèles modulable avec transbordeur, qui incorporait certains éléments existants (pont tournant, portique à combustibles, et sous-stations des épines fortes attenantes). Cette proposition fut retenue, comme on le voit sur le plan (fig. 7) ${ }^{2}$. La réalisation

2- Le Patrimoine des chemins de fer, op. cit., p. 685 ; Le Patrimoine d'Indre-et-Loire, Charenton, Flohic, p. 1216. 


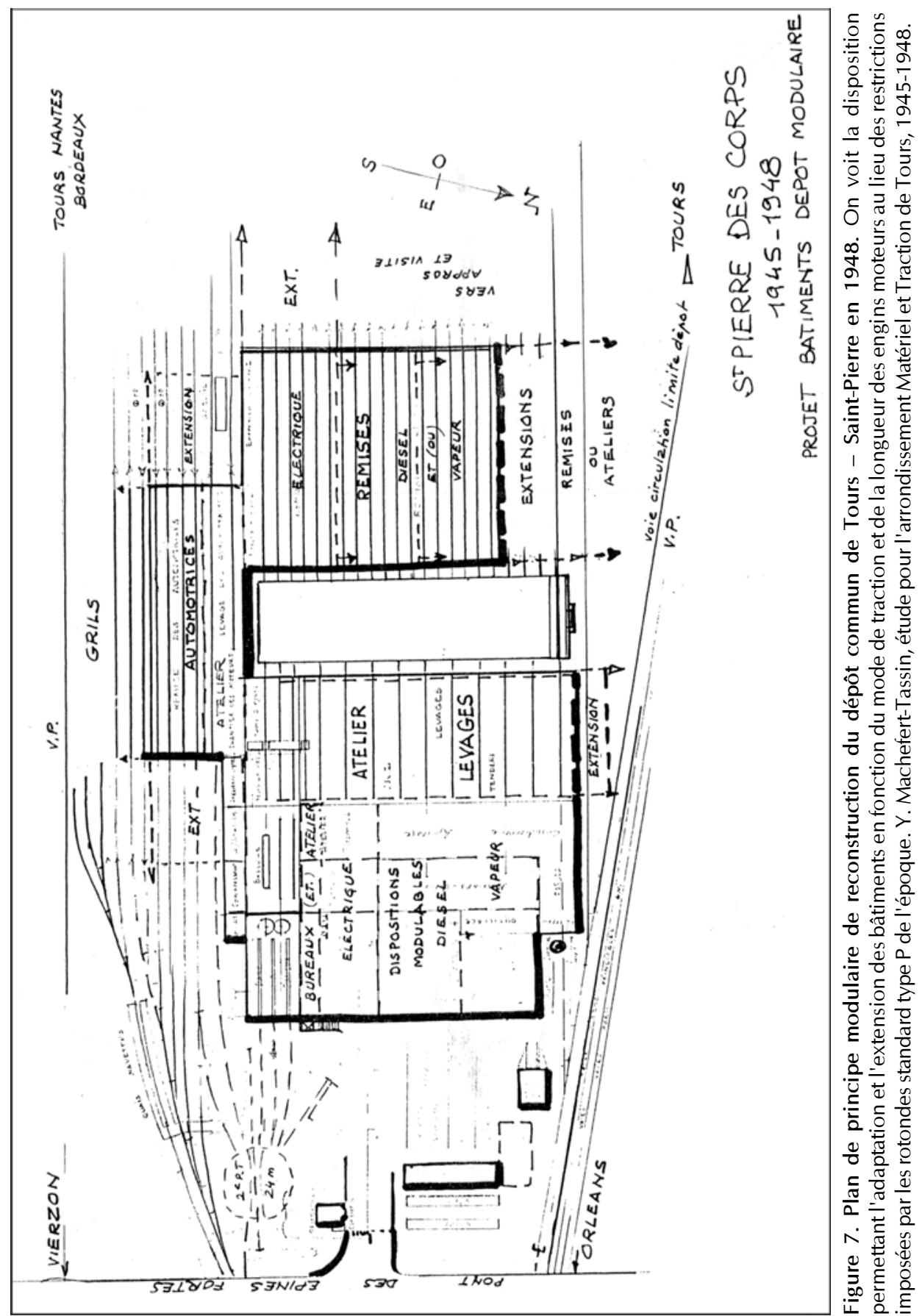


en fut plus modeste certes, avec les incertitudes sur le sort de Tours dont les remises existaient toujours, en exploitation jusqu'en 1957. Cependant il faut reconnaitre que la décision fut prise aussi en considération de plusieurs études précédentes. Tours garda la gare centrale et SaintPierre devint le dépôt commun. La disposition du pont tournant $(23,5 \mathrm{~m})$ put être conservée et les nouvelles remises à transbordeur purent être construites sans gêner le fonctionnement du dépôt à vapeur et des nouveaux grils électriques. Le nouveau dépôt de Saint-Pierre ne put être réalisé complètement qu'en 1958, par le fait de la priorité donnée à la réalisation du dépôt des Aubrais en remplacement de celui d'Orléans.

Curieusement le nouveau Saint-Pierre comporte encore des installations neuves en vapeur, malgré sa mise en service tardive, contrairement aux Aubrais, alors qu'il aurait pu d'emblée n'être qu'un dépôt Diesel et électrique, puisque Vierzon et Nantes conservaient encore des installations (fuel ou charbon) jusqu'en 1960. Certes les relais vapeur étaient nécessaires en attente des locomotives 67000 et 68000 qui arrivèrent en 1967 et remplacèrent les 141 R, 230 F, et $231 \mathrm{G}$ vapeur des années 1960. Les nouvelles installations vapeur n’auront donc été utilisées qu'une douzaine d'années (voir RHCF no18, p. 122). L'électrification de Tours-Nantes et Saumur à Thouars amène ensuite avec le $25 \mathrm{kV}-50 \mathrm{~Hz}$ l'amorce d'un nœud ferroviaire bicourant ne pouvant que s'accroitre en importance et remplacer les engins purement " continu », ce qui amena une trentaine de BB 25500.

Cependant le parc électrique bicourant demeure réduit par comparaison au continu dans l'immédiat après-guerre. De ce fait, l'atelier passe sans transition des levages de locomotives vapeur aux machines Diesel-électrique, le thermique, surtout autorails, prenant la place, en nombre croissant, des locomotives vapeur vouées dès les années 1960 à la disparition. Les réservoirs à fuel lourd demeurèrent encore quelques années pour les $141 \mathrm{R}$ «au mazout» de Nantes et Vierzon en relais, et les parcs à charbon furent les premiers à disparaitre. Par contre un hangar style station-service apparut pour la visite et le ravitaillement des engins Diesel, mais il y eut peu de changements des années 1960 jusqu'au siècle actuel. À noter toutefois la disparition des Aubrais en tant que titulaire d'engins. 


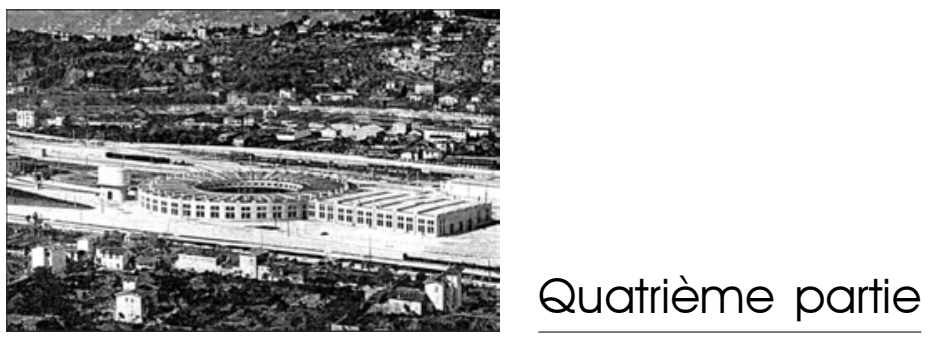

Sociétés cheminotes et communautés urbaines 